\title{
Overexpressed Pseudogene MT1L Associated with Tumor Immune Infiltrates and Indicates a Worse Prognosis in BLCA
}

\section{Yanpeng Ding}

Zhongnan Hospital of Wuhan University https://orcid.org/0000-0002-5081-9431

Nuomin Liu

Zhongnan Hospital of Wuhan University

\section{Mengge Chen}

Zhongnan Hospital of Wuhan University

\section{Yulian Xu}

First People's Hospital of Zaoyang, Hubei

\section{Sha Fang}

Zhongnan Hospital of Wuhan University

\section{Wenbin Xiang}

Zhongnan Hospital of Wuhan University

\section{Xinying Hua}

Zhongnan Hospital of Wuhan University

\section{Gaili Chen}

Zhongnan Hospital of Wuhan University

\section{Yahua Zhong}

Zhongnan Hospital of Wuhan University

Haijun Yu ( $\boldsymbol{D}$ haijunyu@whu.edu.cn )

Zhongnan Hospital of Wuhan University

\section{Research}

Keywords: Pseudogene, Metallothionein, Urinary Bladder Neoplasms, Tumor microenvironment, Prognosis

Posted Date: November 10th, 2020

DOI: https://doi.org/10.21203/rs.3.rs-103098/v1

License: (a) (i) This work is licensed under a Creative Commons Attribution 4.0 International License. 
Version of Record: A version of this preprint was published at World Journal of Surgical Oncology on April 22nd, 2021. See the published version at https://doi.org/10.1186/s12957-021-02231-4. 
3 Yanpeng Ding ${ }^{1 \#}$, Nuomin Liu ${ }^{1 \#}$, Mengge Chen ${ }^{1}$, Yulian $\mathrm{Xu}^{2}$, Sha Fang ${ }^{1}$,

$5 \quad{ }^{1}$ Department of Radiation and Medical Oncology, Hubei Key Laboratory of Tumor

6 Biological Behaviors, Hubei Cancer Clinical Study Center, Zhongnan Hospital of

7 Wuhan University, Wuhan, China, 430071

$8 \quad{ }^{2}$ Department of Oncology, First People's Hospital of Zaoyang, Zaoyang, China, 441200

\section{$9 *$ Correspondence}

Haijun Yu, Wuhan University, Wuhan, 430071, Hubei Province, China, haijunyu@whu.edu.cn

\section{Abstract}

Background BLCA is common cancer worldwide, aggressive, and fatal. Immunotherapy (ICT) has achieved an excellent curative effect in BLCA; however, only part of these patients can benefit from ICT treatment. MT1L belongs to pseudogene, and a previous study has suggested that MT1L can be used as an indicator of prognosis in colorectal cancer. However, the role of MT1L in BLCA has not yet been illuminated.

Methods Data was collected from TCGA, and logistic regression, Kaplan-Meier Plotter, and multivariate Cox analysis have been performed to demonstrate the correlation between pseudogene MT1L and prognosis in BLCA. To identify the association of 
MT1L with tumor-infiltrating immune cells, TIMER and TISIDB were utilized. And GSEA was performed to illuminate the potential biologic function.

Results The expression of MT1L was decreased in BLCA. And it is positively connected with immune cells, like $\operatorname{Tregs}(\rho=0.708)$ and $\operatorname{MDSCs}(\rho=0.664)$. We also confirmed that MT1L is related to typical markers of immune cells, like PD-1, CTLA4. Besides, the high MT1L expression level is related to the high stage of T, N, and the high grade in BLCA and the increased expression of MT1L was significantly associated with the shorter OS of BLCA patients $(\mathrm{P}<0.05)$. Multivariate Cox analysis revealed that MT1L expression could be an independent prognostic factor of BLCA.

Conclusion Collectively, our findings demonstrated that pseudogene MT1L regulates the immune microenvironment, correlates with poor survival, and serves as an independent prognostic biomarker in BLCA.

Keywords Pseudogene, Metallothionein, Urinary Bladder Neoplasms, Tumor microenvironment, Prognosis

\section{Background}

As a heterogeneous disease, bladder cancer is the second most prevalent cancer involving the urinary system, with about 430,000 newly diagnosed cases around the world[1]. Patients with superficial tumors, which account for about $70 \%$, can be treated with transurethral resection of the bladder (TURB), though generally not lifethreatening but have higher risks to confront recurrence[2]. While patients companied 
muscle-invasive have poor prognosis and higher distant metastasis rates[3]. Cisplatinbased systemic chemotherapy remains the first-line treatment for bladder cancer patients with advanced disease and metastatic[4], while the long-term responses are rare, and the recurrence rate is not improving. Besides, half of the MIBC patients are cisplatin-ineligible, and there is no effective standard treatment for these patients[5]. The underlying molecular mechanism of BLCA remains unclear. It is necessary and meaningful to explore the mechanisms of this disease and the factors of its prognosis. MT1L belongs to metallothioneins (MT) [6]. Evidence has shown that MTs could regulate cell growth, proliferation and protect the body from oxidative stress, antineoplastic drugs, and radiation via binding heavy metal, such as zinc/copper[7] [8]. In humans, there are four subtypes of MT MT-2, MT-3, and MT-4 proteins that are encoded by a single gene. MT-1A, $-1 \mathrm{~B},-1 \mathrm{E},-1 \mathrm{~F},-1 \mathrm{G},-1 \mathrm{H},-1 \mathrm{M}$, and $-1 \mathrm{x}$ are active genes belongs to MT-1. And the rest MT-1 genes (MT-1C, -1D, -1I, -1J and -1L) are pseudogenes [9]. Pseudogenes, abundant in the human genome, are a class of IncRNAs that control the expression of their homologous protein-coding genes and do not encode functional proteins due to different types of mutations in their coding sequence. [10]. Recently, accumulating evidence has manifested that pseudogenes play a crucial role in various diseases, especially in human cancers[11]. Several tumor-related pseudogenes have been proved to be indicators in human cancers. For instance, a pseudogene named DUXAP10 is up-regulated in various cancers. It can promote HCC cells' proliferation by activating PI3K/AKT pathway and can be regarded as an independent prognostic biomarker in $\operatorname{HCC}[12,13]$. Besides, some pseudogenes have 
also been proved to contribute to the progression of several cancers, such as breast cancer, gastric cancer, and gallbladder carcinoma[14, 15]. Therefore, the vital functions of pseudogenes in human cancers can’t be ignored.

In the available studies, we first identified a new pseudogene MT1L, of which the expression level, prognosis, and immune-associated of MT1L in BLCA have not been elucidated. Data was collected from TCGA. The association of MT1L with tumorinfiltrating immune cells was demonstrated by the TIMER and TISIDB. Besides, the R-4.0.2, GEPIA, Kaplan-Meier Plotter, UALCAN, and GSEA certified the expression in human cancers and demonstrated the role of MT1L in BLCA. Eventually, the results show us the strong correlation between MT1L and the tumor immune system and the essential prognostic value of MT1L in BLCA.

\section{Material and Methods}

\section{Data acquisition}

Clinical follow-up information and RNA-seq data from 408 BLCA patients were screened out from TCGA [16]; cases with insufficient or missing data on overall survival time, TNM stage, lymph node metastasis were excluded.

\section{GEPIA analysis}

Gene Expression Profiling Interactive Analysis (GEPIA), a web server, analyzes gene expression profiling and interaction in cancer and normal tissues[17]. To confirm if the expression levels of MT1L have changed in different human cancer types and corresponding normal tissues, we used GEPIA to conduct differentially expressed analysis. $\mathrm{P}<0.05$ indicated statistically significant differences. (http://gepia.cancer- 
pku.cn/)

\section{TIMER analysis}

Based on the RNA-seq data, we analyzed the TCGA database via TIMER (Tumor Immune Estimation Resource site) to re-confirm the expression of MT1L in different human cancer types and corresponding normal tissues. After that, as a comprehensive resource for systematic analysis of immune infiltrates, TIMER was applied to measure the correlation between MT1L expression and immune infiltration of BLCA[18]. Meanwhile, we also used it to explore the relationship between MT1L expression and typical immune cells' markers. P-values $<0.05$ were considered statistically significant.

\section{(https://cistrome.shinyapps.io/timer/)}

\section{TISIDB analysis}

TISIDB (tumor-immune system interactions and drug bank database), an integrated repository portal for tumor-immune system interactions, combining five types of data resources to annotate each gene with ten kinds of analysis[19]. In this study, we also used TISIDB to infer the relations between the expression of MT1L and the abundance of immune cells and immunomodulators in BLCA, with $\rho>0.1$ set as the criterion for judging the immune system influenced by MT1L expression.

\section{(http://cis.hku.hk/TISIDB)}

\section{UALCAN analysis}

To acquire the survival curve between different cancers, we first conducted the UALCAN database, a comprehensive and interactive web tool[20]. The student's T-test was employed, and a p-value $<0.05$ was deemed statistically significant. 


\section{(http://ualcan.path.uab.edu)}

\section{Kaplan Meier plotter analysis}

Kaplan Meier plotter, known as a web tool for assessing biomarkers, was performed to analyze the association of MT1L with overall survival (OS) of BLCA patients. This web tool can be used to evaluate the functions of 54,675 genes in 18,674 tumor samples, including breast cancer, ovarian cancer, lung cancer, and gastric cancer[21]. The patient samples were classified into high- and low/medium-expression groups when assessing the prognostic value of MT1L. (www.kmplot.com)

\section{Gene Set Enrichment Analysis (GSEA)}

Gene Set Enrichment Analysis (GSEA) is a powerful analytical method to speculate whether a gene set is enriched in a specific biological state[22]. In this study, it was performed to reveal the critical biological process and relevant signaling pathways affected by the expression level of MT1L. The cut-off criterion was $p<0.05$ and an enrichment score (NES) $>1.5$.

\section{Statistical Analysis by R-4.0.2}

Clinical information was obtained from TCGA and was all conducted by R-4.0.2. Using logistic regression and multivariate $\mathrm{COX}$ regression, we analyzed the correlations between clinicopathological characteristics and MT1L expression and the prognostic value of MT1L in BLCA, respectively. And the correlation heatmap was acquired for the correlation between MT1L and 22 types of immune cells. A p-value < 0.05 was considered statistically significant in this study.

\section{Results}




\section{The mRNA expression levels in BLCA and other human cancers}

Data on the gene expression of 31 types of human cancers were acquired from GEPIA and TIMER. Results show in Fig.1A and Fig.1B, both databases showed consistent results, with low MT1L expression among BLCA, BRCA, CHOL, COAD, KIRP, LIHC, LUAD, LUSC, READ, STAD, and THCA. Eventually, MT1L was identified as lowly expressed in BLCA.

\section{Strong Correlation between MT1L and Tumor Immune Infiltration in BLCA}

Independent tumor-infiltrating lymphocytes are essential in the prediction of the overall survival rate. Thus, we analyzed the correlation between MT1L expression and 22 types of immune cells based on the 408 samples downloaded from the TCGA database by R-4.0.2. As Fig.2A exhibited, B cell plasma, naive CD4 T cells, CD4 memory $\mathrm{T}$ cells activated, $\mathrm{T}$ follicular helper cells, regulatory $\mathrm{T}$ cell, gamma delta $\mathrm{T}$ cells, NK cells resting, monocyte, macrophages M0, macrophages M1, macrophages M2, myeloid dendritic cells activated and neutrophil are the main immune cells affected by MT1L expression.

TIMER was used to further identify the correlation between MT1L and immune infiltration in BLCA. CD8 T cells $(r=0.328, p<0.001)$, CD4 T cells $(r=0.242, p<0.001)$, neutrophil $(r=0.342, \mathrm{p}<0.001)$ and dendritic cells $(r=0.448, \mathrm{p}<0.001)$ are significantly correlated with MT1L expression (Fig.2B).

TISIDB database was used to evaluate the spearman correlation between MT1L expression and lymphocyte and immunomodulators (Fig.2C-F). Results display a strong correlation between MT1L expression and TILs, such as effector memory CD8 
$\mathrm{T}$ cell $($ Tem-CD8) $(\rho=0.549, \quad \mathrm{p}<2.2 \mathrm{e}-16)$, central memory CD8 $\mathrm{T}$ cell (TcmCD8) $(\rho=0.493, p<2.2 \mathrm{e}-16), \mathrm{T}$ follicular helper cell $(\mathrm{Tfh})(\rho=0.619, p<2.2 \mathrm{e}-16)$, regulatory T cell $($ Treg $)(\rho=0.708, p<2.2 \mathrm{e}-16)$, natural killer T cell $(\mathrm{NKT})(\rho=0.683$, $\mathrm{p}<2.2 \mathrm{e}-16), \quad$ myeloid-derived suppressor $\operatorname{cell}(\operatorname{MDSC})(\rho=0.664, \quad \mathrm{p}<2.2 \mathrm{e}-16)$. Furthermore, it is obvious that the correlation between MT1L expression and TILs is the strongest in BLCA than in other human cancers. Immunomodulators can be further classified into immunoinhibitors, immunostimulators, and $\mathrm{MHC}$ molecules. For immunoinhibitors, MT1L expression exhibits a strong correlation with CSF1R, HAVCR2, IL10, PDCD1, and PDCD1LG2. For immunostimulators, MT1L is significantly correlated with CD28, CD70, CD86, IL2RA, IL6, TNFRSF4, and

TNFRSF8. For MHC, HLA-B, HLA-DMB, HLA-DPB1, HLA-DQA1, HLA-DQB1, and HLA-DRB1 show strong pertinence with MT1L expression.

Therefore, results in all three databases show that MT1L is an essential and especial factor in the tumor immune microenvironment, especially in BLCA.

\section{Correlation between MT1L and Immune Cell Type Markers.}

The relationship between MT1L expression and typical immune cell markers was explored through the TIMER database (Table1). We found a significant positive correlation of MT1L with CD8+T cell markers, T cell (general) markers(CD3E, CD2),

173 monocyte marker (CD86), natural killer cell markers(KIR2DL3, KIRIDL4), dendritic 174 cell markers ( HLA-DPB1, HLA-DQB1, HLA-DPA1) and functional T cells markers, 175 including Th1 marker (IFNG, TNF), Treg markers (ENTPD1) and T cell exhaustion 176 (PD-1, CTLA4). Notably, we noticed MT1L exhibited strong correlations with makers 
177 of monocyte and M2 macrophage, which means that MT1L may regulate macrophage 178 polarization in BLCA.

Of note are the strong correlations among MT1L with dendritic cell markers (NRP1, ITGAX). It has been proved that DCs can promote tumor metastasis by increasing Treg cells and reducing $\mathrm{CD}^{+} \mathrm{T}$ cell cytotoxicity[23]. And strong correlations can't be ignored among MT1L expression with T cell exhaustion and Treg markers, such as LAG3, TIM-3, GZMB, FOXP3, IL2RA, and TGFB1. Studies show that FOXP3, as a vital factor, restrains cytotoxic T cells from attacking tumor cells[24]. In addition, the correlations among MT1L with $\mathrm{T}$ cell exhaustion markers mean that high expression of MT1L in BLCA leads to exhaustion of T cells. Therefore, the results above confirm the important role of MT1L in immune escape in the bladder cancer microenvironment.

(Table 1 is placed at the end of the document text file.)

\section{Correlation Between MT1L Expression and Clinicopathological Characteristics} not yet characterized, so we performed GEPIA and R-4.0.2 based on the data downloaded from the TCGA database to analyze the MT1L expression level and its correlation between clinicopathological characteristics in BLCA. The results in Fig1 revealed that MT1L expression in BLCA tumor samples is lower than in normal samples. Then we evaluated the correlation between MT1L expression and several clinical characteristics of BLCA patients. 408 BLCA samples from the TCGA database 
were included in our analysis. Using logistic regression, we found that MT1L has excellent diagnostic value in BLCA patients. The MT1L expression level significantly 201 correlated with patients age $(p=0.039)$, weight $(p=0.01)$, race (Asian vs other, $p=0.043$;

202 Asian vs White, $\mathrm{p}<0.001)$, N (N0 vs $>\mathrm{N} 0, \mathrm{p}=0.026)$, stage (I-II vs III, $\mathrm{p}<0.001$; I-II vs 203 IV, $\mathrm{p}<0.001), \mathrm{T}(<\mathrm{T} 2$ vs $\mathrm{T} 3, \mathrm{p}<0.001 ;<\mathrm{T} 2$ vs $\mathrm{T} 4, \mathrm{p}=0.001)$, grade (high vs low, 204 $\mathrm{p}=0.003$ ), as shown in Table2.

Table 2 logistic regression analysis

\begin{tabular}{|c|c|c|c|}
\hline Clinical characteristics & $\mathbf{N}$ & OR & p-Value \\
\hline Age & 408 & $1.02(1.00-1.04)$ & 0.039 \\
\hline Gender & 408 & $0.76(0.48-1.18)$ & 0.216 \\
\hline Weight & 408 & $1.01(1.00-1.02)$ & 0.01 \\
\hline \multicolumn{4}{|l|}{ Race } \\
\hline Asian vs Other & 67 & $3.01(1.06-9.21)$ & 0.043 \\
\hline Asian vs White & 369 & $5.56(2.63-13.20)$ & $<0.001$ \\
\hline Other vs White & 348 & $0.80(0.33-1.87)$ & 0.605 \\
\hline M (M0 vs M1) & 207 & $2.95(0.83-13.80)$ & 0.118 \\
\hline N (NO vs >N0) & 366 & $0.61(0.40-0.94)$ & 0.026 \\
\hline \multicolumn{4}{|l|}{ Stage } \\
\hline I-II vs III & 272 & $2.53(1.56-4.14)$ & 0 \\
\hline I-II vs IV & 266 & $2.50(1.54-4.12)$ & 0 \\
\hline III vs IV & 274 & $1.06(0.66-1.70)$ & 0.81 \\
\hline \multicolumn{4}{|l|}{$\mathbf{T}$} \\
\hline$<\mathrm{T} 2$ vs $\mathrm{T} 3$ & 317 & $2.26(1.43-3.60)$ & 0 \\
\hline$<\mathrm{T} 2$ vs $\mathrm{T} 4$ & 181 & $3.03(1.59-5.98)$ & 0.001 \\
\hline T3 vs T4 & 252 & $1.11(0.62-2.01)$ & 0.728 \\
\hline Grade (high vs low) & 405 & $0.153(0.04-0.46)$ & 0.003 \\
\hline
\end{tabular}

Table 2 The relationship between MTIL and clinical characteristics in BLCA, analyzed 
by logistic regression with 408 samples obtained from TCGA.

\section{The Clinical Value of MT1L in BLCA}

We conducted the UALCAN database and acquired the survival curve of BLCA

(Fig.3A). Results revealed that the higher expression of MT1L in BLCA indicates the worse overall survival time among 406 BLCA patients $(n=406, p=0.0023)$.

Kaplan Meier plotter was performed to confirm further the correlation between MT1L expression and the OS (Fig.3B). As the pictures show, the increased expression of MT1L in BLCA indicates a shorter overall survival time among 404 patients $(n=404$, $\mathrm{HR}=1.58,95 \% \mathrm{CI}=1.15-2.18, \mathrm{p}=0.0044)$. As the same, high expression of MT1L in bladder carcinoma forebodes an unfavorable prognosis.

Premised on the correlation between MT1L expression and the prognosis, we then conducted a multivariate cox analysis. In this analysis, MT1L exhibited its significant prognostic value in BLCA. Tumor stage, person neoplasm status, and MT1L expression 221 are verified independent prognostic factors, respectively (Table 3).

Table 3 Multivariate Cox regression analysis

\begin{tabular}{lcc}
\hline \multicolumn{1}{c}{ Clinical characteristics } & HR $(95 \% \mathrm{CI})$ & p-value \\
\hline Gender & $1.288(0.861-1.926)$ & 0.218 \\
Weight & $0.999(0.990-1.228)$ & 0.875 \\
$\mathrm{~T}$ & $1.411(1.080-1.843)$ & $0.012^{*}$ \\
Grade & $0.768(0.177-3.324)$ & 0.724 \\
Person Neoplasm status & $3.814(2.640-5.509)$ & $9.51 \mathrm{E}-13^{* * *}$ \\
MTIL & $1.087(1.001-1.180)$ & 0.048 \\
\hline
\end{tabular}


characteristics factors.

Gene sets enriched in BLCA expression phenotype.

GSEA is a powerful analytical method to speculate whether a gene set is enriched in a specific biological state[22], was performed to identify the key biological process and relevant signaling pathways affected by the expression level of MT1L. (Table 4). The GO enrichment analysis showed that MT1L was related to the production of interleukin $1,6,8,17$. It is all known that interleukins are critical factors in regulating immune cell. Meanwhile, the results also revealed that MT1L plays a role in regulating immune cells like $\mathrm{T}$ cell, B cell, macrophage, and lymphocyte, consistent with the previous results. Besides, CTLA4-Pathway was also enriched. All those results reconfirmed that MT1L probably plays an integral correlative role in tumor immunity.

236 Another important thing is that MT1L was closely related to bladder cancer, involving 237 tumorigenesis, invasiveness, and recurrence, which suggests a potentially crucial biological function role of MT1X in BLCA.

\begin{tabular}{lccc}
\hline \multicolumn{1}{c}{ NAME } & Enrichment & NES & p \\
& Score & & \\
\hline GO_CHRONIC_INFLAMMATORY_RESPONSE & 0.667 & 1.651 & 0.009 \\
GO_INTERLEUKIN_17_PRODUCTION & 0.631 & 1.682 & 0.014 \\
GO_INTERLEUKIN_6_PRODUCTION & 0.523 & 1.578 & 0.037 \\
GO_INTERLEUKIN_1_PRODUCTION & 0.511 & 1.547 & 0.05 \\
GO_INTERLEUKIN_8_PRODUCTION & 0.555 & 1.539 & 0.035 \\
GO_MACROPHAGE_CHEMOTAXIS & 0.607 & 1.662 & 0.03 \\
GO_MACROPHAGE_DIFFERENTIATION & 0.576 & 1.558 & 0.04 \\
\hline
\end{tabular}




\begin{tabular}{lccc}
\hline GO_REGULATION_OF_MACROPHAGE_CHEMOTAXIS & 0.629 & 1.656 & 0.025 \\
GO_REGULATION_OF_MONONUCLEAR_CELL_MIGRATION & 0.641 & 1.647 & 0.025 \\
GO_REGULATION_OF_B_CELL_MEDIATED_IMMUNITY & 0.629 & 1.585 & 0.039 \\
GO_POSITIVE_REGULATION_OF_T_CELL_PROLIFERATION & 0.619 & 1.671 & 0.045 \\
GO_REGULATION_OF_LYMPHOCYTE_MIGRATION & 0.569 & 1.598 & 0.03 \\
BIOCARTA_CTLA4_PATHWAY & 0.779 & 1.605 & 0.034 \\
LINDGREN_BLADDER_CANCER_HIGH_RECURRENCE & 0.67 & 1.749 & 0.014 \\
LINDGREN_BLADDER_CANCER_CLUSTER_2B & 0.661 & 1.862 & 0 \\
OSMAN_BLADDER_CANCER_UP & 0.57 & 1.645 & 0.025 \\
ANASTASSIOU_MULTICANCER_INVASIVENESS_SIGNATURE & 0.784 & 1.718 & 0.012 \\
WANG_TUMOR_INVASIVENESS_UP & 0.533 & 1.666 & 0.01 \\
CROMER_TUMORIGENESIS_UP & 0.645 & 1.665 & 0.031 \\
\hline
\end{tabular}

Table 4 The key biological process and relevant signaling pathways affected by the expression level of MT1L analyzed by GESA. Gene sets with NES>1.5 and p-value < 0.05 are considered as significant.

\section{Discussion}

MT1L is a pseudogene expressed in multiple cancer tissues, including the brain, breast, thyroid, pancreas, etc. [25]. It has been confirmed that MT1L can be used as an 247 indicator of prognosis in colorectal cancer[26]. However, there is limited literature on 248 the potential biological impact of MT1L in BLCA, so we conducted the first-ever 249 analysis study.

250 Here, we observed that the expression of MT1L in BLAC is lower than that of 251 normal bladder tissue. Logistic regression revealed the high MT1L expression related 252 to the high $\mathrm{T}, \mathrm{N}$, and high grades. Therefore, we reasonably speculate that MT1L is 253 related to the tumors' malignant biological behavior. Besides, the high expression of 13 
MT1L was significantly associated with the shorter overall survival time of BLCA patients $(\mathrm{p}<0.05)$. And multivariate Cox analysis showed that MT1L expression could be an independent prognostic factor of BLCA.

The most interesting discovery is that in BLCA, diverse immune cell surface markers and immune infiltration levels are associated with MT1L expression. Recently, ICT has changed the intervention measures for urinary cancer (including advanced bladder cancer) [27]. ICT has achieved a good curative effect in BLCA; however, it is undeniable that only part of these patients can benefit from ICT treatment. As a highly immunogenic malignant tumor, TME immune response disorder in BLCA plays a decisive role in tumors' occurrence and development; there are still many problems to be solved about the immunotherapy for BLCA. It is urgent to find effective therapeutic targets that affect the level of immune cell infiltration. This study indicates a correlation between MT1L expression and the infiltration of TILs. Among these 30 kinds of tumors, the correlation between MT1L expression and TILs in BLCA showed the reddest color, that means the strongest correlation. The same result can also be found in the correlation between MT1L expression and immunoregulatory molecules.

Particularly, BLCA is characterized by the marked infiltration with immune cells such as Tregs and MDSCs [28, 29]. Tregs and MDSCs are key regulators of antitumor responses in BLCA, and the intra-tumoral presence of those are correlated with the poor clinical outcome has already been established [30, 31]. Tregs serve as a vital immunosuppressive factor to promote tumor occurrence and development, inhibit antitumor immunity, and help in tumor immune escape [32]. Likewise, MDSCs are 
widely distributed and have strong immunosuppressive activity in the TME that inhibit cytotoxic T cells' proliferation and activation, leading to the failure of the antitumor immune response and promoting cancer progression and chemoresistance [33]. TISIDB analysis indicated a substantial positive connection of MT1L expression with Tregs' infiltration levels $(\rho=0.708)$ and $\operatorname{MDSCs}(\rho=0.664)$ in BLCA. Therefore, a significant conclusion can be drawn that the high MT1L expression is associated with high immune infiltration levels in BLCA. Thus, we suggested that MT1L has a potential influence on tumor immunology and plays a crucial role in regulating tumor immunity.

Likewise, the relationships among immune cell surface markers and MT1L expression implicate the significant meanings of MT1L in regulating the tumor immune microenvironment of BLCA. According to the TIMER, the expression of MT1L was positively correlated with $\mathrm{T}$ cell depletion markers (PD-1, CTLA4). Under normal conditions, the interaction of PD-L1/PD-1 can inhibit the proliferation of CD8+T cells and the cytotoxic function mediated by $\mathrm{T}$ cell receptor (TCR), thus avoiding autoimmunity and resolve inflammation [27]. The combination of PD-L1 and T cell surface receptor PD-1 leads to T cells' dysfunction, which contributes to the failure of T cells [34]. In BLCA, the high expression of MT1L is positively correlated with the high infiltration level of immune cells; meanwhile, it also indicates poor survival and prognosis. That seems contradictory. However, the relationship between MT1L expression and $\mathrm{T}$ cell depletion markers provides a reasonable explanation for it.

Also, MT1L has a strong correlation with the markers of tumor-associated macrophages (TAM) and M2 macrophages, especially the cell surface markers of M2 
type macrophages. TAM is an essential part of TME, plays a vital role in the occurrence and development of tumors by mediating immunosuppression, promotes tumor proliferation, metastasis, and angiogenesis, and even participates in tumor drug resistance [35-37]. TAM can differentiate into M1 macrophage or M2 macrophage phenotype. Clinical research showed that cancer patients' poor prognosis is significantly correlated with the number of M2 macrophages [38]. The strong correlation between MT1L expression and M2 macrophage surface markers in our explains the potential mechanism of poor prognosis in BLCA patients with high expression of MT1L. Thereby, targeting MT1L may reduce the infiltration of TAM, can be a new thought for immunotherapy.

Another meaningful aspect of this study is discovering the correlation between MT1L and interleukin displayed in our GSEA analysis. Interleukin (IL) 17 is produced by a subset of T-helper (Th) cells with a strong effect on immune cells and plays an important role in cancer pathogenesis by directly or indirectly interacting with tumors.

315 It has been studied to confirm that the changing patterns of IL-17's expression might 316 be associated with the infiltration of inflammatory cells, which might also contribute to 317 the occurrence and development in BLCA [39, 40]. Reduced IL-17 can be used as an 318 indicator for following the course and immune response to cancer in BLCA [41]. As our results revealed, MT1L expression was closely related to interleukin, which may 
contribute to MT1L probably plays an integral correlative role in TME. However, the

321 role of MT1L in other tumors needs further study.

322

\section{Conclusion}

In this study, we constructed a series of analyses to estimate the role of MT1L in the tumor immune system and its prognosis value in BLCA. It is reasonable to conclude that high MT1L expression correlates with a negative prognosis in BLCA. Meanwhile, it is also associated with different immune cells, such as T cells, Tregs, and MDSCs.

Consequently, MT1L has a vital influence on immune infiltration and can be used as an independent prognostic indicator of BLCA. We hope to provide an additional feasible method for evaluating the prognosis and a valuable new target for antitumor immunotherapy in BLCA. Nonetheless, further research on MT1L in BLCA needs to be done.

(Table 1 should be placed on line 189 of the text.)

Table1 Correlation analysis between MT1L and relate genes and makers of immune cells in TIMER in BLCA

\begin{tabular}{|c|c|c|c|c|c|}
\hline \multirow[b]{3}{*}{ Description } & \multirow[b]{3}{*}{ Gene markers } & \multicolumn{4}{|c|}{ BLCA } \\
\hline & & \multicolumn{2}{|c|}{ None } & \multicolumn{2}{|c|}{ Purity } \\
\hline & & Cor & $P$ & Cor & $P$ \\
\hline \multirow[t]{2}{*}{ CD8+T cell } & CD8A & 0.447 & 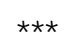 & 0.227 & $\star * \star$ \\
\hline & CD8B & 0.395 & $\star \star \star ~$ & 0.249 & $\star * *$ \\
\hline \multirow[t]{3}{*}{ T cell(general) } & CD3D & 0.438 & 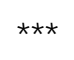 & 0.185 & $* *$ \\
\hline & CD3E & 0.487 & 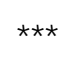 & 0.215 & 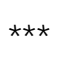 \\
\hline & CD2 & 0.479 & $* * *$ & 0.214 & $* * *$ \\
\hline \multirow[t]{2}{*}{ B cell } & CD19 & 0.331 & $\star * \star *$ & 0.078 & .14 \\
\hline & CD79A & 0.381 & $\star \star \star *$ & 0.106 & * \\
\hline \multirow[t]{2}{*}{ Monocyte } & CD86 & 0.622 & $\star \star \star *$ & 0.418 & $\star \star *$ \\
\hline & CD115(CSF1R) & 0.674 & 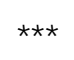 & 0.494 & $\star * \star$ \\
\hline \multirow[t]{3}{*}{ TAM } & CCL2 & 0.558 & 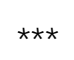 & 0.347 & $\star * *$ \\
\hline & CD68 & 0.431 & 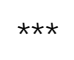 & 0.251 & $* * *$ \\
\hline & IL10 & 0.602 & 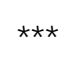 & 0.431 & $\star * *$ \\
\hline M1 macrophage & INOS(NOS2) & 0.106 & $\star *$ & 0.055 & .30 \\
\hline
\end{tabular}




\begin{tabular}{|c|c|c|c|c|c|}
\hline & COX2(PTGS2) & 0.134 & $* \star$ & 0.026 & .62 \\
\hline \multirow[t]{3}{*}{ M2 macrophage } & CD163 & 0.654 & $* \star *$ & 0.461 & $\star * \star$ \\
\hline & VSIG4 & 0.674 & $* * *$ & 0.505 & $\star \star \star *$ \\
\hline & MS4A4A & 0.648 & 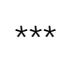 & 0.455 & $\star \star \star \star ~$ \\
\hline \multirow[t]{2}{*}{ Neutrophils } & CD11b(ITGAM) & 0.591 & $\star \star \star$ & 0.364 & $\star \star \star \star ~$ \\
\hline & CD66b(CEACAM8) & 0.008 & .87 & 0.036 & .49 \\
\hline \multirow[t]{7}{*}{ Natural killer cell } & KIR2DL1 & 0.226 & $* \star \star$ & 0.071 & .18 \\
\hline & KIR2DL3 & 0.393 & 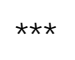 & 0.248 & $\star \star \star *$ \\
\hline & KIR2DL4 & 0.375 & $\star \star \star$ & 0.222 & 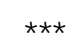 \\
\hline & KIR3DL1 & 0.281 & 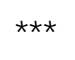 & 0.169 & * \\
\hline & KIR3DL2 & 0.282 & 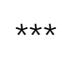 & 0.132 & * \\
\hline & KIR3DL3 & 0.111 & * & 0.062 & .24 \\
\hline & KIR2DS4 & 0.273 & $* * *$ & 0.133 & * \\
\hline \multirow[t]{5}{*}{ Dendritic cell } & HLA-DPB1 & 0.497 & 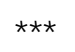 & 0.235 & 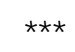 \\
\hline & HLA-DQB1 & 0.472 & $\star * \star *$ & 0.243 & 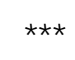 \\
\hline & HLA-DPA1 & 0.450 & 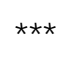 & 0.207 & $\star \star \star *$ \\
\hline & BDCA-4(NRP1) & 0.541 & 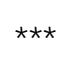 & 0.456 & 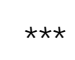 \\
\hline & CD11c(ITGAX) & 0.620 & $\star \star \star *$ & 0.392 & $* \star *$ \\
\hline \multirow[t]{5}{*}{ Th1 } & T-bet(TBX21) & 0.436 & 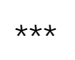 & 0.190 & $\star \star$ \\
\hline & STAT4 & 0.565 & $* * *$ & 0.361 & $\star \star \star *$ \\
\hline & STAT1 & 0.366 & $\star \star \star *$ & 0.187 & $\star \star *$ \\
\hline & IFN- $\gamma($ IFNG $)$ & 0.393 & 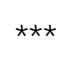 & 0.219 & $\star \star \star \star ~$ \\
\hline & TNF- $\alpha(T N F)$ & 0.384 & $\star \star \star *$ & 0.244 & $* \star *$ \\
\hline \multirow[t]{4}{*}{ Th2 } & GATA3 & -0.489 & 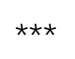 & -0.402 & $\star \star \star *$ \\
\hline & STAT6 & -0.263 & $* * *$ & -0.215 & $\star \star \star *$ \\
\hline & STAT5A & 0.237 & $\star * *$ & 0.034 & .52 \\
\hline & IL13 & 0.202 & $\star \star \star$ & 0.036 & .49 \\
\hline \multirow[t]{4}{*}{ Treg } & FOXP3 & 0.214 & $\star \star * *$ & 0.433 & $\star \star \star \star ~$ \\
\hline & IL2RA & 0.606 & $* \star *$ & 0.395 & $\star * *$ \\
\hline & CD39(ENTPD1) & 0.457 & $\star \star \star$ & 0.247 & $\star \star \star *$ \\
\hline & TGF $\beta(T G F B 1)$ & 0.444 & $\star \star \star *$ & 0.375 & $\star \star \star *$ \\
\hline \multirow[t]{5}{*}{ T cell exhaustion } & PD-1(PDCD1) & 0.475 & $* \star *$ & 0.248 & 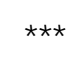 \\
\hline & CTLA4 & 0.511 & $* * *$ & 0.294 & $\star \star \star *$ \\
\hline & LAG3 & 0.519 & $\star \star \star$ & 0.332 & 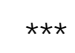 \\
\hline & TIM-3(HAVCR2) & 0.621 & $\star \star \star *$ & 0.410 & 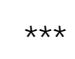 \\
\hline & GZMB & 0.533 & $* * *$ & 0.328 & $\star \star \star *$ \\
\hline
\end{tabular}

Table 1 The relationship between MT1L expression and typical markers of immune cells by TIMER database $(* P<0.05, \quad * * P<0.01, \quad * * * P<0.001)$.

338 Abbreviation BLCA: Bladder cancer; MDSCs: Myeloid-derived suppressor cells;

339 Tregs: Regulatory T cells; TAMs: Tumor-associated macrophages; OS: Overall 18 
LUAD: Lung adenocarcinoma; LUSC: Lung squamous cell carcinoma; READ:

\section{References}

1. Butt SU, Malik L: Role of immunotherapy in bladder cancer: past, present and future. Cancer Chemother Pharmacol 2018, 81:629-645.

2. Antoni S, Ferlay J, Soerjomataram I, Znaor A, Jemal A, Bray F: Bladder Cancer Incidence and Mortality: A Global Overview and Recent Trends. Eur Uro/ 2017, 71:96-108.

6. Cherian MG, Jayasurya A, Bay BH: Metallothioneins in human tumors and potential roles in carcinogenesis. Mutat Res 2003, 533:201-209. Arriaga JM, Levy EM, Bravo Al, Bayo SM, Amat M, Aris M, Hannois A, Bruno L, Roberti MP,

3. Kaufman DS, Shipley WU, Feldman AS: Bladder cancer. Lancet 2009, 374:239-249.

4. Nadal R, Bellmunt J: Management of metastatic bladder cancer. Cancer Treat Rev2019,

5. Rouanne M, Roumiguié $M$, Houédé N, Masson-Lecomte A, Colin P, Pignot G, Larré S, Xylinas E, Rouprêt M, Neuzillet Y: Development of immunotherapy in bladder cancer: present and future on targeting PD(L)1 and CTLA-4 pathways. World J Urol 2018, 36:1727-1740.

8. Ruttkay-Nedecky B, Nejdl L, Gumulec J, Zitka O, Masarik M, Eckschlager T, Stiborova M, Loria FS, et al: Metallothionein expression in colorectal cancer: relevance of different isoforms for tumor progression and patient survival. Hum Patho/ 2012, 43:197-208.

Adam V, Kizek R: The role of metallothionein in oxidative stress. Int J Mol Sci 2013, 14:6044-6066.

9. Krizkova S, Kepinska M, Emri G, Eckschlager T, Stiborova M, Pokorna P, Heger Z, Adam V: An insight into the complex roles of metallothioneins in malignant diseases with emphasis on (sub)isoforms/isoforms and epigenetics phenomena. Pharmacology \& therapeutics 2018, 183.

0. Milligan MJ, Harvey E, Yu A, Morgan AL, Smith DL, Zhang E, Berengut J, Sivananthan J, Subramaniam R, Skoric A, et al: Global Intersection of Long Non-Coding RNAs with Processed and Unprocessed Pseudogenes in the Human Genome. Front Genet 2016, 7:26.

11. Xiao-Jie L, Ai-Mei G, Li-Juan J, Jiang X: Pseudogene in cancer: real functions and 
promising signature. J Med Genet 2015, 52:17-24.

12. Yue C, Ren Y, Ge H, Yan L, Xu Y, Wang G, Wu J: Pseudogene DUXAP10 can be used as a diagnostic and prognostic biomarker in human cancers. J Cell Physiol 2019, 234:23685-23694.

13. Yue C, Liang C, Ge H, Yan L, Xu Y, Li G, Li P, Wei Z, Wu J: Pseudogene DUXAP10 acts as a diagnostic and prognostic marker and promotes cell proliferation by activating PI3K/AKT pathway in hepatocellular carcinoma. Onco Targets Ther 2019, 12:45554566.

14. Lv W, Wang L, Lu J, Mu J, Liu Y, Dong P: Downregulation of TPTE2P1 Inhibits Migration and Invasion of Gallbladder Cancer Cells. Chem Biol Drug Des 2015, 86:656-662.

15. Zheng L, Li X, GU Y, Ma Y, Xi T: Pseudogene CYP4Z2P 3'UTR promotes angiogenesis in breast cancer. Biochemical and biophysical research communications 2014, 453:545-551.

16. Blum A, Wang P, Zenklusen JC: SnapShot: TCGA-Analyzed Tumors. Ce// 2018, 173:530.

17. Tang Z, Li C, Kang B, Gao G, Li C, Zhang Z: GEPIA: a web server for cancer and normal gene expression profiling and interactive analyses. Nucleic Acids Res 2017, 45

18. Li T, Fan J, Wang B, Traugh N, Chen Q, Liu JS, Li B, Liu XS: TIMER: A Web Server for Comprehensive Analysis of Tumor-Infiltrating Immune Cells. Cancer Res 2017, 77:e108-e110.

19. Ru B, Wong CN, Tong Y, Zhong JY, Zhong SSW, Wu WC, Chu KC, Wong CY, Lau CY, Chen I, et al: TISIDB: an integrated repository portal for tumor-immune system interactions. Bioinformatics (Oxford, England) 2019, 35:4200-4202.

20. Chandrashekar DS, Bashel B, Balasubramanya SAH, Creighton CJ, Ponce-Rodriguez I, Chakravarthi B, Varambally S: UALCAN: A Portal for Facilitating Tumor Subgroup Gene Expression and Survival Analyses. Neoplasia 2017, 19:649-658.

21. Gyorffy B, Lánczky A, Szállási Z: Implementing an online tool for genome-wide validation of survival-associated biomarkers in ovarian-cancer using microarray data from 1287 patients. Endocrine-related cancer 2012, 19:197-208.

22. Subramanian A, Tamayo P, Mootha VK, Mukherjee S, Ebert BL, Gillette MA, Paulovich A, Pomeroy SL, Golub TR, Lander ES, Mesirov JP: Gene set enrichment analysis: a knowledge-based approach for interpreting genome-wide expression profiles. Proc Natl Acad Sci U S A 2005, 102:15545-15550.

23. Sawant A, Hensel JA, Chanda D, Harris BA, Siegal GP, Maheshwari A, Ponnazhagan S: Depletion of plasmacytoid dendritic cells inhibits tumor growth and prevents bone metastasis of breast cancer cells. J Immuno/ 2012, 189:4258-4265.

24. Facciabene A, Motz GT, Coukos G: T-regulatory cells: key players in tumor immune escape and angiogenesis. Cancer Res 2012, 72:2162-2171.

25. Holloway AF, Stennard FA, West AK: Human metallothionein gene MT1L mRNA is present in several human tissues but is unlikely to produce a metallothionein protein. FEBS Lett 1997, 404:41-44.

26. Hung KC, Huang TC, Cheng CH, Cheng YW, Lin DY, Fan JJ, Lee KH: The Expression Profile and Prognostic Significance of Metallothionein Genes in Colorectal Cancer. Int J Mol Sci2019, 20.

27. Wu Z, Liu J, Dai R, Wu S: Current status and future perspectives of immunotherapy in bladder cancer treatment. Science China Life sciences 2020. 
28. Eruslanov E, Neuberger M, Daurkin I, Perrin GQ, Algood C, Dahm P, Rosser C, Vieweg J, Gilbert SM, Kusmartsev S: Circulating and tumor-infiltrating myeloid cell subsets in patients with bladder cancer. Int J Cancer 2012, 130:1109-1119.

29. Sjödahl G, Lövgren K, Lauss M, Chebil G, Patschan O, Gudjonsson S, Månsson W, Fernö $\mathrm{M}$, Leandersson $\mathrm{K}$, Lindgren $\mathrm{D}$, et al: Infiltration of $\mathrm{CD}^{+}$and $\mathrm{CD}^{+} 8^{+}$cells in bladder cancer is subtype specific and affects the outcome of patients with muscle-invasive tumors. Urologic oncology 2014, 32:791-797.

30. Maeda S, Murakami K, Inoue A, Yonezawa T, Matsuki N: CCR4 Blockade Depletes Regulatory T Cells and Prolongs Survival in a Canine Model of Bladder Cancer. Cancer immunology research 2019, 7:1175-1187.

31. Yang G, Shen W, Zhang Y, Liu M, Zhang L, Liu Q, Lu HH, Bo J: Accumulation of myeloidderived suppressor cells (MDSCs) induced by low levels of IL- 6 correlates with poor prognosis in bladder cancer. Oncotarget 2017, 8:38378-38388.

32. Li C, Jiang P, Wei S, Xu X, Wang J: Regulatory T cells in tumor microenvironment: new mechanisms, potential therapeutic strategies and future prospects. Mol Cancer 2020, 19:116

33. Groth C, Hu X, Weber R, Fleming V, Altevogt P, Utikal J, Umansky V: Immunosuppression mediated by myeloid-derived suppressor cells (MDSCs) during tumour progression. Br J Cancer 2019, 120:16-25.

34. Alsaab HO, Sau S, Alzhrani R, Tatiparti K, Bhise K, Kashaw SK, lyer AK: PD-1 and PD-L1 Checkpoint Signaling Inhibition for Cancer Immunotherapy: Mechanism, Combinations, and Clinical Outcome. Frontiers in pharmacology 2017, 8:561.

35. Pinton L, Masetto E, Vettore M, Solito S, Magri S, D’Andolfi M, Del Bianco P, Lollo G, Benoit J-P, Okada $\mathrm{H}$, et al: The immune suppressive microenvironment of human gliomas depends on the accumulation of bone marrow-derived macrophages in the center of the lesion. Journal for ImmunoTherapy of Cancer 2019, 7.

36. Qian B-Z, Pollard JW: Macrophage Diversity Enhances Tumor Progression and Metastasis. Ce//2010, 141:39-51.

37. Komohara Y, Takeya M: CAFs and TAMs: maestros of the tumour microenvironment. The Journal of pathology 2017, 241:313-315.

38. Murray Peter J, Allen Judith E, Biswas Subhra K, Fisher Edward A, Gilroy Derek W, Goerdt S, Gordon S, Hamilton John A, Ivashkiv Lionel B, Lawrence T, et al: Macrophage Activation and Polarization: Nomenclature and Experimental Guidelines. Immunity 2014, 41:14-20.

39. Liu Y, Yang W, Zhao L, Liang Z, Shen W, Hou Q, Wang Z, Jiang J, Ying S: Immune analysis of expression of IL-17 relative ligands and their receptors in bladder cancer: comparison with polyp and cystitis. BMC Immuno/ 2016, 17:36.

40. Wang L, Yi T, Kortylewski M, Pardoll DM, Zeng D, Yu H: IL-17 can promote tumor growth through an IL-6-Stat3 signaling pathway. The Journal of experimental medicine 2009, 206:1457-1464.

41. Baharlou R, Ahmadi Vasmehjani A, Dehghani A, Ghobadifar MA, Khoubyari M: Reduced interleukin-17 and transforming growth factor Beta levels in peripheral blood as indicators for following the course of bladder cancer. Immune network 2014, 14:156163. 


\section{Declaration}

\section{Ethics approval and consent to participate}

This was not applicable to this manuscript.

\section{Consent for publication}

Consent for publication was obtained from all participants.

\section{Availability of data and materials}

This was not applicable to this manuscript.

\section{Competing interest}

All the authors, including Yanpeng Ding, Nuomin Liu, Mengge Chen, Yulian Xu, Sha Fang, Wenbin Xiang, Xinying Hua, Gaili Chen, Yahua Zhong, and Haijun Yu have no conflict of interest.

\section{Funding}

This research was supported by National Natural Science Foundation [No. 82073349], Zhongnan Hospital of Wuhan University, Science, Technology and Innovation Seed Fund [No. ZNPY2016043]; Natural Science Foundation of Hubei Province [Grant No. 2018CFB561] and Scientific Fund of Health Commission of Hubei Province [Grant No. WJ2019M264].

\section{Authors' Contributions}

Haijun Yu designed, revised and finalized the manuscript. Yanpeng Ding and Nuomin Liu was the first author who contributed to drafting and editing of the manuscript. Mengge Chen and Yulian Xu prepared the figures and tables. Sha Fang and Wenbin Xiang contributed to literature search. Xinying Hua and Gaili Chen participated in the drafting and editing manuscript. Yahua Zhong participated in the revision and 
487 coordination. All authors read and approved the final manuscript.

488 Acknowledgements Not applicable

\section{Figure Legends:}

490 Figure 1 Expression analysis of MT1L in different tumor tissues.(A) MTL1 mRNA level in 31

491 types of human cancers analyzed by GEPIA (B)MT1L mRNA level analyzed by

$492 \quad \operatorname{TIMER}(* \mathrm{P}<0.05, * * \mathrm{P}<0.01, * * * \mathrm{P}<0.001)$

493 Figure 2 MT1L expression was related to immune infiltration. (A) Correlation between

494 MT1L expression and 22 types of immune cells conducted by R-4.0.2 $(\mathrm{p}<0.001)$. (B)

495 Correlation between MT1L expression and immune cells analyzed by TIMER. (C-F)

496 Relations among the abundance of TILs(C), immunoinhibitors(D),

497 immunostimulators(E), and $\mathrm{MHC}(\mathrm{F})$, top 2 are displayed, respectively.

498 Figure 3 The survival curve acquired from (A) UALCAN database and (B) Kaplan499 Meier plotter. The red line represents the high expression level of MT1L, and the other 500 represents the low expression. 


\section{Figures}
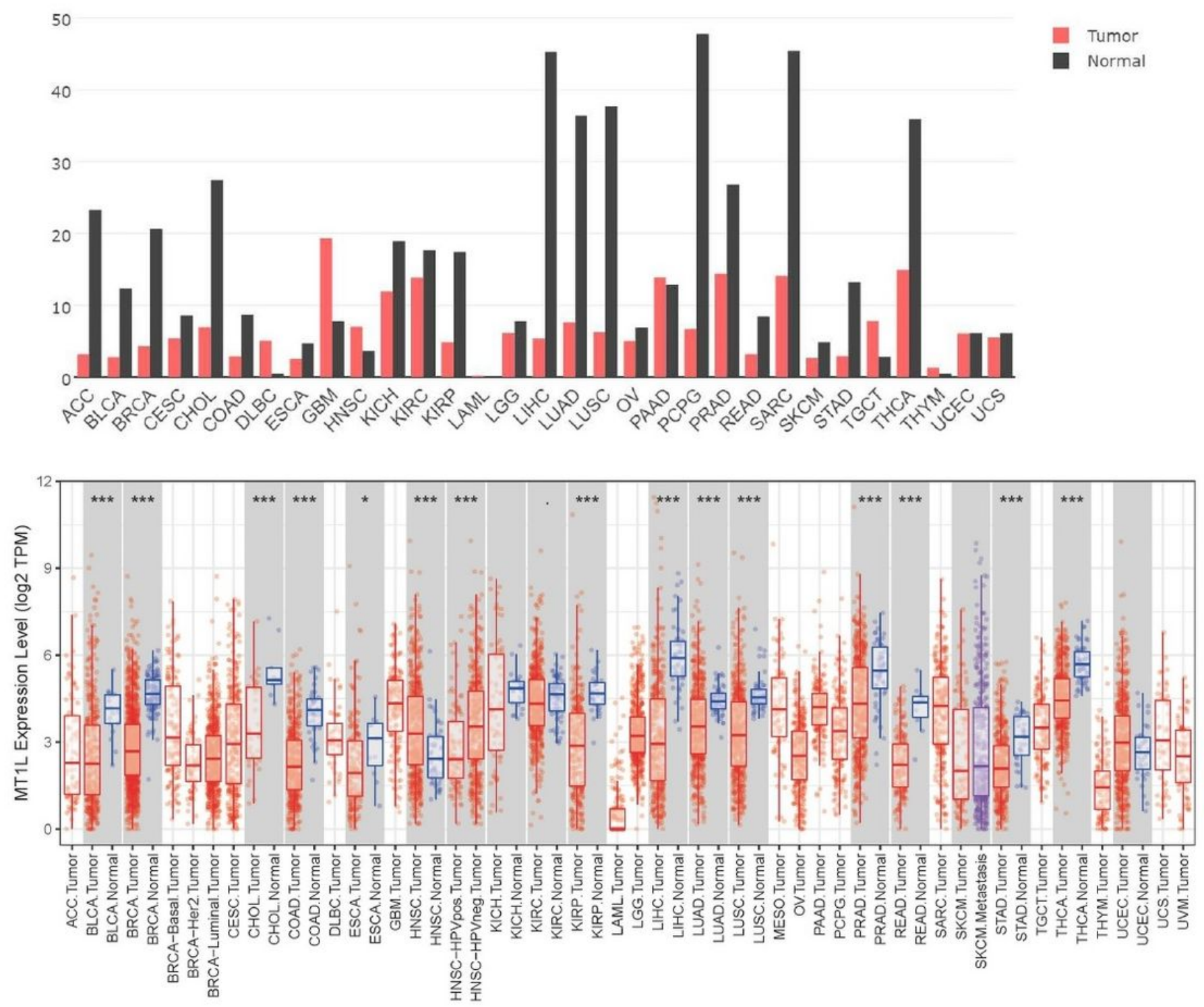

\section{Figure 1}

Expression analysis of MT1L in different tumor tissues.(A) MTL1 mRNA level in 31 types of human cancers analyzed by GEPIA (B)MT1L mRNA level analyzed by TIMER $\left({ }^{*} P<0.05 \nabla^{\star \star *} P<0.01{ }^{\star}{ }^{\star \star \star} P<0.001\right)$ 

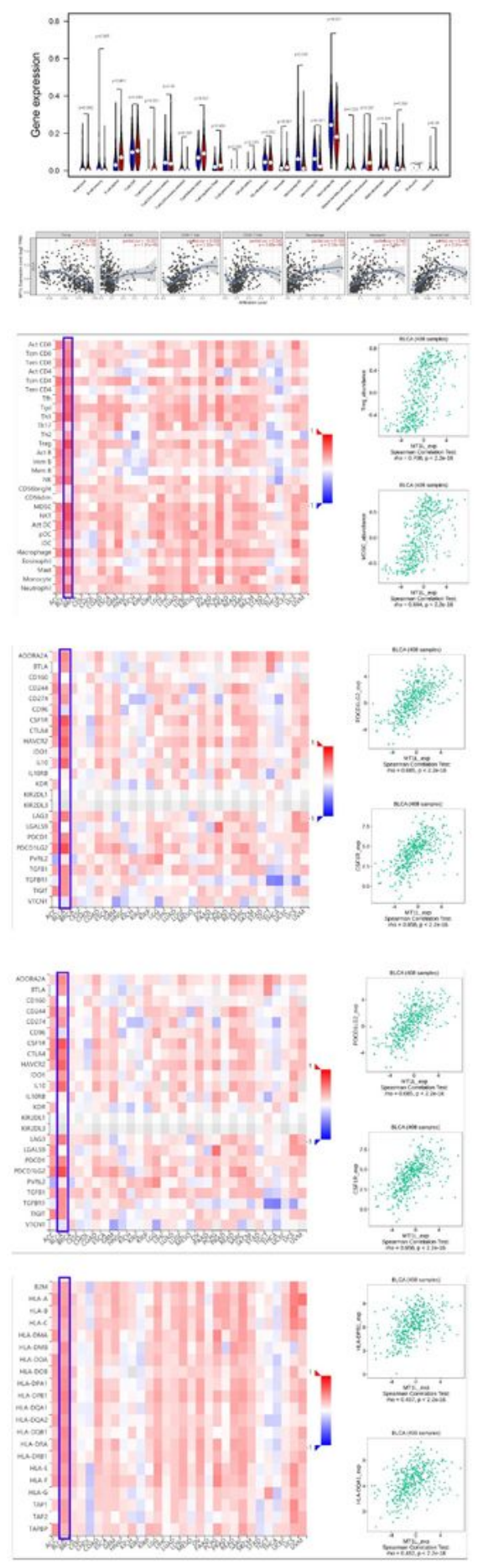

\section{Figure 2}

MT1L expression was related to immune infiltration. (A) Correlation between MT1L expression and 22 types of immune cells conducted by R-4.0.2( $p<0.001)$. (B) Correlation between MT1L expression and immune cells analyzed by TIMER. (C-F) Relations among the abundance of TILs(C), immunoinhibitors(D), immunostimulators(E), and $\mathrm{MHC}(\mathrm{F})$, top 2 are displayed, respectively. 

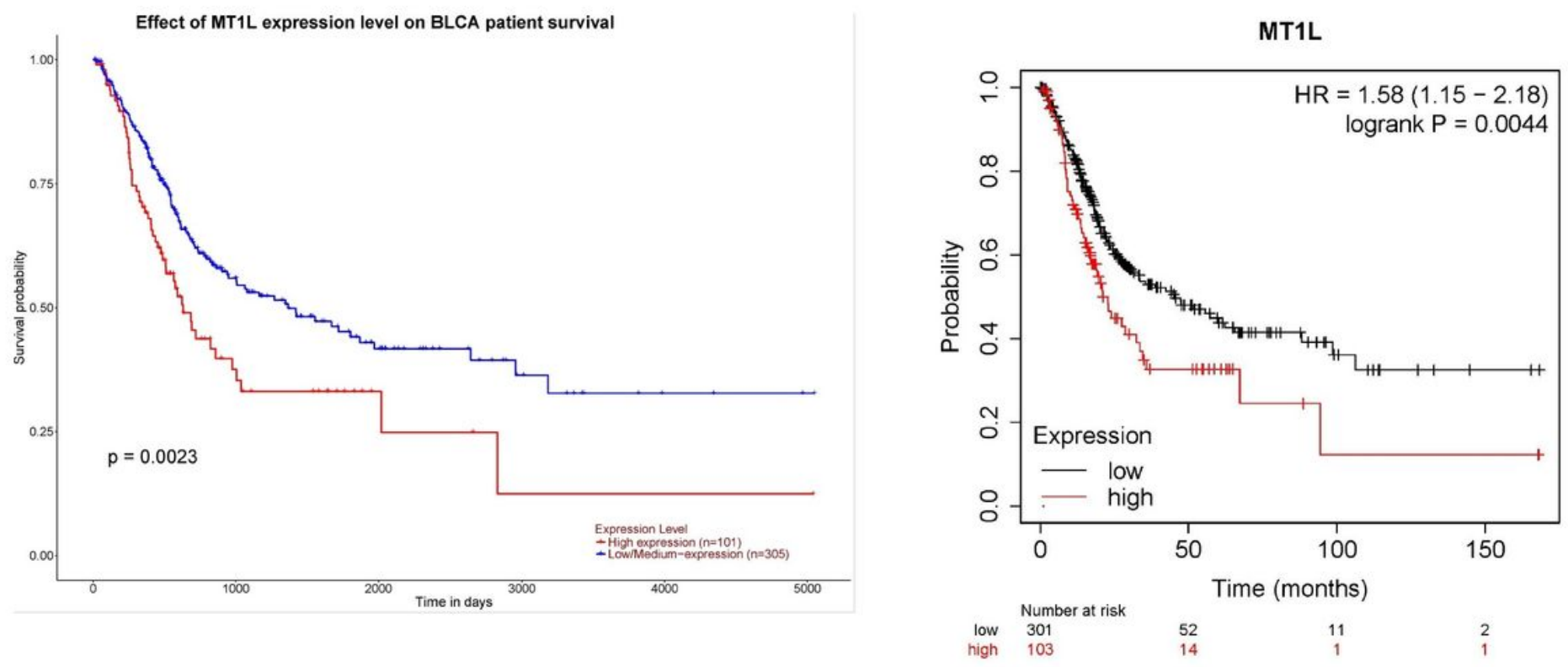

Figure 3

The survival curve acquired from (A) UALCAN database and (B) Kaplan-Meier plotter. The red line represents the high expression level of MT1L, and the other represents the low expression. 